\title{
Stabilité \\ des pentes sous-marines de l'atoll de Mururoa sous sollicitations dynamiques
}

J. BOUCHEZ

Y. CARISTAN

C. MARIOTTI

Département d'Analyse

et Surveillance

de l'Environnement.

Commissariat à l'Énergie

atomique, BP 12

91680 Bruyères-le-Châtel
La stabilité des pentes océaniques de l'atoll de Mururoa a fait l'objet d'un suivi tout au long de l'exploitation du Centre d'Expérimentation du Pacifique français. La zone Nord de la couronne corallienne a été instrumentée après la constatation au début des années 80 de la réactivation de certaines físsures bordières. La présence de calcaires crayeux tendres dans les formations de pente est à l'origine de ces físsures bordières naturelles. Les sollicitations dynamiques dues aux essais ont entraîné un début de fluage des calcaires crayeux. L'analyse en laboratoire des propriétés mécaniques de ces roches, la réalisation de maquettes analogiques et de simulations numériques ont permis de comprendre le comportement de ces matériaux de pente au cours de la réalisation de plus de 80 essais. Leur évolution compactante laisse prévoir une évolution à long terme du mouvement vers une stabilisation.

\section{Stability of the fore-reef of Mururoa atoll under dynamic loading}

The stability of the ocean slopes of the Muroroa atoll has been monitored throughout the operation of the French Centre d'Expérimentation du Pacifique. The Northern zone of the corallian rim has been instrumented in the early eighties wher the reactivation of some border cracks was verified. The soft chalky limestone present in the slope formations caused these natural border cracks. The dynamic loads resulting from the tests initiated a creeping motion of the chalky limestones. The analysis in laboratory of the mechanical properties of these rocks, the creation of analog scale models and numerical simulations contribued to the understanding of the slope materials behavior during more than 80 tests. The trend to compacting mechanical behaviour of the chalky limestones indicates that the long term evolution of the motion is probably toward stabilization. 


\section{Introduction}

Les pentes sous-marines de talus continentaux, de zones côtières ou même de pentes abyssales faibles ont été l'objet de déstabilisations qui ont pu avoir des conséquences néfastes pour les biens et les personnes vivant dans les régions affectées (Voight, 1978). De tels glissements ont eu lieu sur les pentes sous-marines au niveau du port de Nice en 1979 (Habib, 1994), à proximité du port de Skagway en Alaska en 1994 (Kulikov et al., 1996), dans la baie de Kitimat au Canada en 1975 (Murty, 1979). Des glissements sous-marins d'une dimension largement supérieure et engendrant des courants de turbidité ont été également observés sur les flancs des îles Hawaï (Moore, 1964), sur les marges continentales norvégiennes (Jansen et al., 1987), au Canada sur les Grands Bancs à la suite d'un séisme (Heezen et Ewing, 1952). Des références bien documentées concernant les problèmes de stabilité des pentes sous-marines sous sollicitation sismique étant rares, il a semblé opportun de rapporter ici les données et la connaissance des mécanismes liés au comportement mécanique des formations carbonatées de la zone Nord de l'atoll de Mururoa, en particulier sous sollicitation sismique. En effet, cette zone du Centre d'expérimentation nucléaire a été suivie pendant plus de quinze ans, période durant laquelle environ une cen- taine d'essais ont été réalisés. Depuis dix ans, son comportement a été évalué de façon prévisionnelle avant chaque essai, et suivi a posteriori.

\section{2}

\section{Le contexte géomécanique naturel}

L'atoll de Mururoa est constitué d'un soubassement volcanique vieux de plus de dix millions d'années. Il est surmonté d'un chapeau de roches calcaires d'origine corallienne, résultat de l'installation des coraux autour du volcan dès que la diminution de son activité l'a permis. L'éloignement progressif du point chaud qui lui a donné naissance et l'augmentation de son propre poids au cours de la construction ont entraîné la subsidence graduelle de l'édifice (Buigues et al,, 1992; Guille et al, 1992; Bouchez et Lecomte, 1995): Aujourd'hui, seule une couronne corallienne étroite émerge, enserrant un lagon peu profond (maximum $50 \mathrm{~m}$ ). Du côté de l'océan les pentes plongent en moyenne à $45^{\circ}$ vers le plancher océanique qui se trouve à environ $3500 \mathrm{~m}$ de profondeur aux alentours immédiats (Fig. 1).

La géométrie des atolls de Mururoa et Fangataufa, stade ultime de l'évolution du volcan vieux et éteint, reflète celle qu'il avait dans sa jeunesse lorsque se sont édifiées les constructions coralliennes il y a plus de dix millions d'années. En effet, ces constructions coralliennes s'édifient très rapidement après l'émersion du jeune vol-

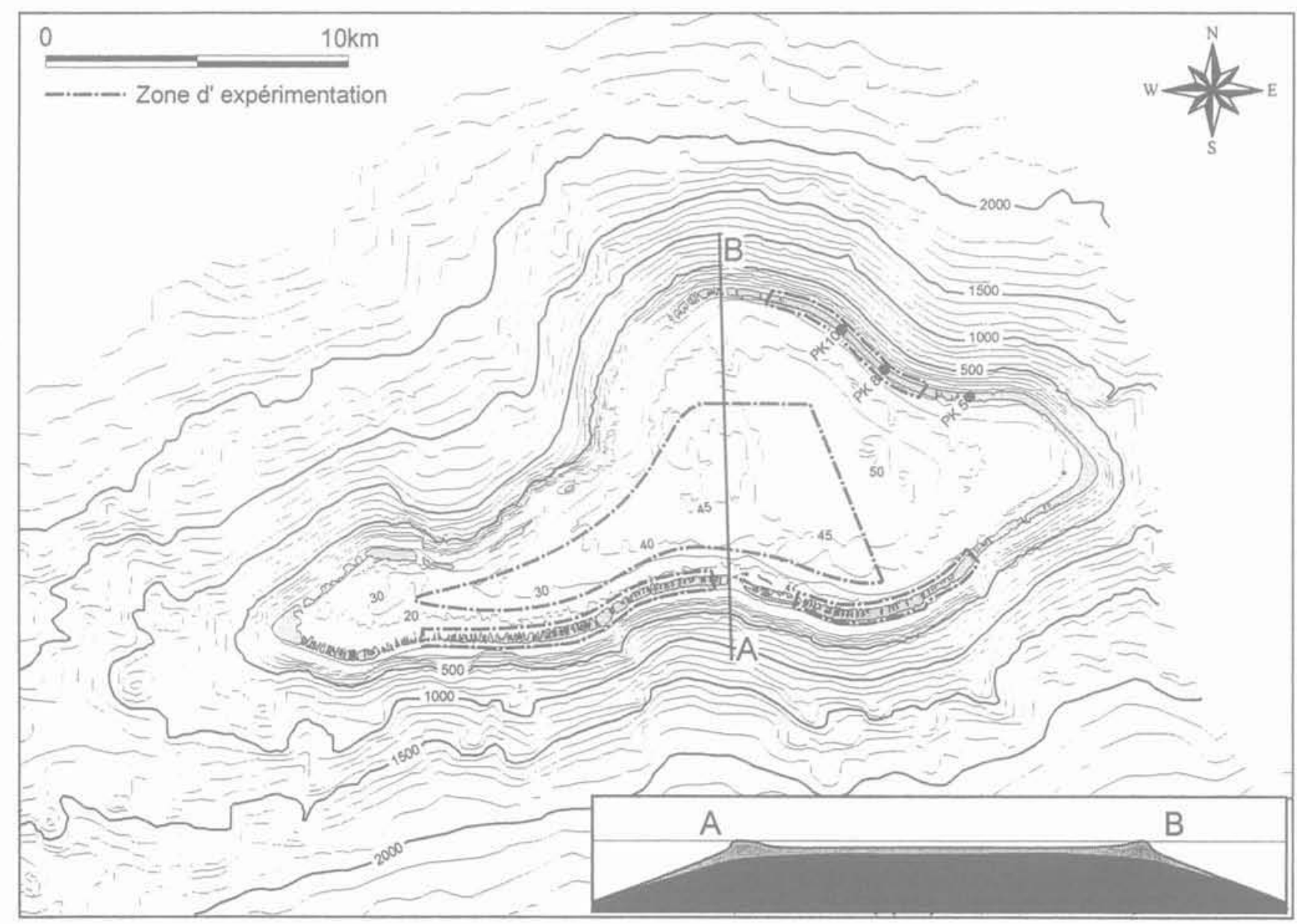

FIG.1 Bathymétrie et coupe de l'atoll de Mururoa. En gris foncé, le socle basaltique large et assis sur des fonds océaniques de -2500 à -3500 mètres. En clair, le chapeau de roches coralliennes.

Bathymetry and cross-section of the Mururoa atoll. Dark gray : the basalt shelf, broad and seated on 2,500 to 3,500 meter sea floors. Light gray: the corallian rock cap. 
can, dès que son activité a suffisamment décru pour les laisser se développer. Elles en fossilisent la morphologie, modelée par les glissements qui ont pu se produire au moment de la pleine activité. Ainsi retrouve-t-on inscrite dans la courbure concave des côtes la marque des anciennes zones de glissement du soubassement volcanique, stabilisées depuis l'installation des coraux.

Sur ce substratum stable, la croissance des coraux, horizontalement vers l'océan ou verticalement, s'est faite en fonction de l'histoire du niveau des océans et des climats. La structure géologique des flancs et le profil de la pente externe résultent directement de cette histoire. Ainsi trouve-t-on sur la figure 2, surmontant le socle volcanique, des couches horizontales de calcaires coralliens et de dolomies plus ou moins indurées avec des surfaces karstiques (Buigues, 1996), Par ailleurs, il en résulte une organisation latérale des formations de pente qui est très caractéristique d'un atoll. On notera en particulier l'existence de calcaires crayeux où se trouvent des structures sédimentaires de dissolution liées à la circulation centripète des fluides océaniques au sein des masses rocheuses carbonatées. Au niveau de la pente océanique et sur ces formations crayeuses, se trouvent des formations détritiques classiques. Enfin, juste en contact avec le substratum volcanique, on notera la présence de dépôts argileux, appelés zone de transition, et provenant de l'érosion du volcan lorsqu'il était encore émergé. Le profil général de la pente correspond au profil d'équilibre des différentes formations. Vers $120 \mathrm{~m}$ des encoches marquent le niveau de la mer tel qu'il était lors de la dernière glaciation. Un tel profil se retrouve sur d'autres atolls comme par exemple à Mayotte dans l'océan Indien. C'est un bon reflet géomorphologique de l'histoire climatique du globe.

Les caractéristiques géotechniques de ces différentes formations sont données au tableau I. Elles résultent essentiellement de mesures effectuées en laboratoire et sont utilisées dans les modélisations numériques effectuées pour l'étude de la stabilité des pentes. L'analyse du champ de contraintes purement statique, obtenu par simulation numérique bidimensionnelle, permet de définir la surface naturelle présentant le coefficient de sécurité minimal. La surface potentielle de glissement est totalement dans les car- bonates. En surface se créent des zones de traction. Effectivement, on observe sur une grande partie du pourtour de la couronne des deux atolls une fracturation bordière ancienne, scellée par un ciment carbonaté, correspondant à l'existence tout à fait classique de contraintes en traction localisées essentiellement au niveau du tombant. Elles se sont vraisemblablement développées au cours des temps géologiques au fur et à mesure de la consolidation des formations carbonatées, en particulier des calcaires crayeux.

\section{3}

\section{La réaction mécanique dynamique des massifs}

\section{1}

\section{Les observations}

\subsection{1 \\ Le tassement en surface}

Toute observation et, a fortiori, toute mesure de tassement suppose d'avoir un point de référence, origine des mesures de nivellement, et d'être sûr de sa stabilité. Dans le cas d'une île océanique isolée, cela peut être résolu aujourd'hui par mesure GPS. Ce n'était pas le cas il y a vingt ans lors des premiers essais souterrains. Seule la mesure du niveau moyen de l'océan pouvait donner une indication de la stabilité relative de ce point de référence. Encore faut-il prendre en compte et éliminer toutes les évolutions du niveau moyen liées aux variations de pression atmosphérique. C'est ce qui a été fait à partir des données marégraphiques de Mururoa dont la séquence est presque continue depuis 1975. L'évolution du niveau moyen est de $3,5 \mathrm{~mm} / \mathrm{an}$ environ. Le même traitement donne une montée de la mer de $2,5 \mathrm{~mm}$ aux îles Gambier et $7 \mathrm{~mm}$ à Tahiti. Plus généralement cet ordre de grandeur correspond à une variation très lente et millimétrique du niveau de tous

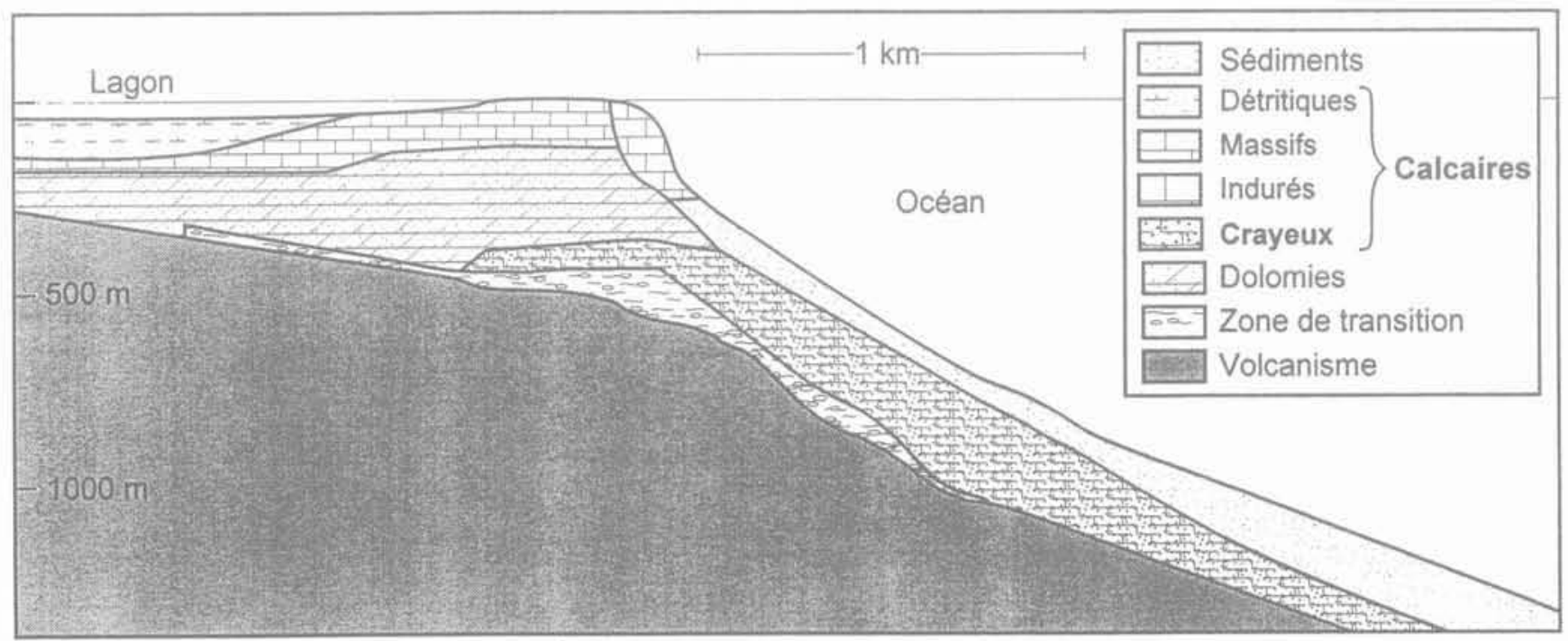

FiG. 2 Coupe du bord océanique du chapeau de roches coralliennes. On remarquera en particulier les calcaires crayeux très développés. Cross-section of the ocean edge of the corallian rock cap. Note the highly developed chalky limestone. 
TABLEAUI Propriétés moyennes des matéraiux constituant les flancs de l'atoll de Mururoa. Mean properties of the materials making up the flanks of the Mururoa atoll.

\begin{tabular}{|c|c|c|c|c|c|c|c|}
\hline Matériaux & $\begin{array}{l}\text { Densité p } \\
\left(\mathrm{kg} / \mathrm{m}^{3}\right)\end{array}$ & $\begin{array}{c}\text { Module } \\
\text { d'Young E } \\
\left(10^{10} \mathrm{~Pa}\right)\end{array}$ & $\begin{array}{l}\text { Coefficient } \\
\text { de Poisson v }\end{array}$ & $\begin{array}{l}\text { Vs } \\
(\mathrm{m} / \mathrm{s})\end{array}$ & $\begin{array}{c}V p \\
(\mathrm{~m} / \mathrm{s})\end{array}$ & $\begin{array}{c}\text { Cohésion C } \\
\left(10^{\circ} \mathrm{Pa}\right)\end{array}$ & $\begin{array}{c}\text { Angle } \\
\text { de frottement } \\
\varphi \text { (degres) }\end{array}$ \\
\hline Calcaíres indurés & 2400 & 1.45 & 0,4 & 1468 & 3598 & 50 & 35 \\
\hline Calcaires détritiques & 2050 & 0,6 & 0,3 & 1060 & 1984 & 15 & 45 \\
\hline Calcaires massifs & 2250 & 1,4 & 0,3 & 1546 & 2894 & 40 & 25 \\
\hline Dolomie sous lagon & 2200 & 1,1 & 0,3 & 1386 & 2594 & 40 & 25 \\
\hline Dolomie sous couronne & 2450 & 1,74 & 0,4 & 1592 & 3901 & 50 & 35 \\
\hline Calcaires crayeux & 2000 & 0.1 & 0,3 & 1250 & 2350 & 5 & 30 \\
\hline Zone de transition & 2200 & 1,1 & 0,3 & 1386 & 2594 & 10 & 30 \\
\hline Sédiments & 2150 & 0,44 & 0,4 & 855 & 2094 & 10 & 40 \\
\hline Volcanisme & 2350 & 2,9 & 0.2 & 2267 & 3702 & 80 & 55 \\
\hline
\end{tabular}

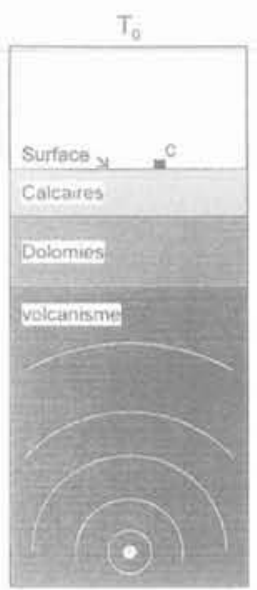

Explosion

Acceleration (q)

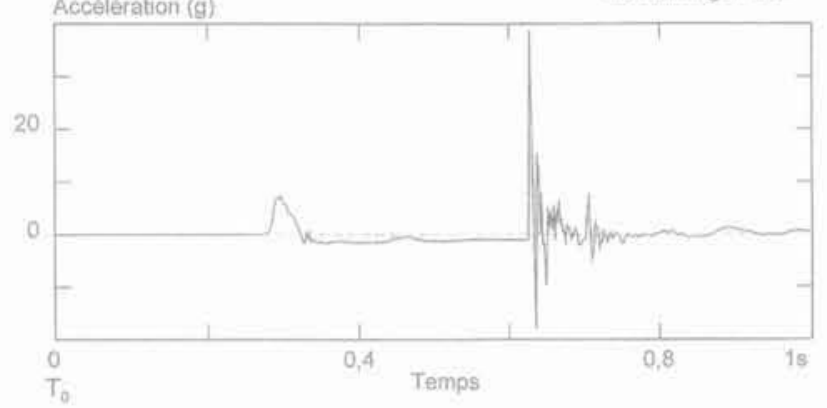

FiG.3 Décomposition du phénomène de tassement superficiel instantané à l'aplomb d'un essai souterrain. Signal enregistré par le capteur en surface. Decomposition of the instantaneous superficial subsidence phenomenon plumb with an underground test. Signal recorded by a surface sensor.

les océans de la planète entre 1930 et 1980 (Douglas, 1991). On peut donc considérer que le point de référence pour le nivellement utilisé à Mururoa est resté techniquement stable au cours des vingt dernières années.

La réalisation d'essais dans l'atmosphère s'est terminée dès 1974 au profit d'essais souterrains sous la couronne. Parmi tous les effets mécaniques immédiats se produisant lors d'un essai, le tassement de la couronne corallienne à la verticale du point zéro a fait l'objet d'un suivi particulier. Il est lié au phénomène de réflexion à la surface libre du sol de l'onde de pression provenant du point zéro. En effet, l'onde réfléchie se combinant avec l'onde incidente engendre des contraintes en traction à quelques mètres de la surface libre, qui provoquent la rupture en traction de la roche et le départ en vol balistique de la dalle de surface (Fig. 3). C'est le phénomène d'écaillage. Lors de sa retombée, elle provoque un choc local dit choc d'écaillage qui compacte les roches carbonatées plus ou moins bien cimentées se trouvant sur les quelques dizaines de mètres les plus superficiels. Les lois phénoménologiques établies au Centre d’Expérimentation du Pacifique permettent de prévoir, en fonction des caractéristiques de l'explosion, l'amplitude des tassements; de l'ordre de quelques dizaines de centimètres sauf en quelques endroits. Ils ne présentent pas d'évolution notable en fonction du temps sauf dans deux zones bien circonscrites (PK 8 et PK 10) du Nord de l'atoll oủ les tassements, après une forte composante immédiate, ont présenté une évolution faible en fonction du temps, mais mesurable par les moyens de nivellement de grande précision utilisés (Fig. 4).

\section{9}

\section{Observations du comportement mécanique de la zone Nord de l'atoll de Mururoa}

\section{- Les premières observations de surface}

Certains essais, effectués dans les années 1979-1980 sous la partie Nord de la couronne corallienne, ont entraîné un tassement immédiat important, une réactivation des failles bordières anciennes et l'apparition d'un jeu de fractures superficielles sur la dalle corallienne de surface. Ce n'est que quelques années plus tard que l'observation des tassements en fonction du temps a mis en évidence une évolution faible mais continue des formations de flanc de cette zone qui n'était plus utilisée pour les expérimentations. Le tassement le plus important est observé entre les points kilométriques 7 et 9 (PK 7 et PK 9), centré sur la zone dite 

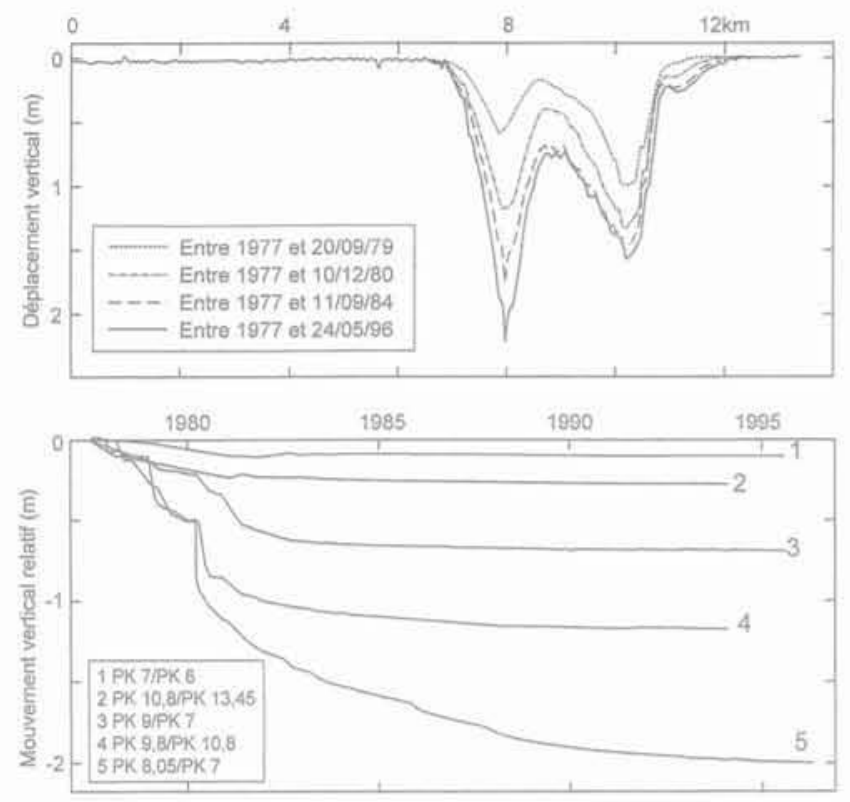

FIG.4 En haut: évolution des profils de tassement de la zone Nord de la couronne corallienne entre 1977 et 1996. En bas: évolution temporelle relative entre différents points de la couronne Nord. Top: evolution of the subsidence profiles in the Northern area of the corallian crown between 1977 and 1996. Bottom: relative evolution in time between various points of the Northern rim.
Camélia (Fig. 5). Deux autres zones présentant les mêmes symptômes ont été surveillées. Il s'agit d'une zone située au niveau du PK 10 appelée "Françoise», et d'une autre située au niveau du PK 5 appelée «Irène $»$. Dans les deux premiers cas, des essais souterrains avaient été réalisés à l'aplomb de ces zones. Dans le dernier cas, aucun essai n'a été réalisé à moins de $2,5 \mathrm{~km}$.

Des repères planimétriques de précision ont été installés afin de mesurer la composante horizontale du mouvement telle qu'elle se révèle à travers l'existence de la fracturation superficielle en traction du platier. Ils confirment une composante horizontale dirigée vers l'océan (Fig. 6). Dès lors, une instrumentation des couches profondes a été décidée. Elle permet de mesurer à la fois la déformation des formations carbonatées, le comportement du soubassement volcanique et le suivi de la microsismicité dans cette zone. Elle vient compléter dans la troisième dimension les observations faites en surface.

\section{- Les observations de profondeur}

L'instrumentation installée est décrite sur la figure 7. Elle a pour objectif à la fois le suivi de l'évolution à long terme et l'alerte en cas de mouvement rapide. Deux types de mesures sont effectuées:

- des mesures sismiques, avec des capteurs en surface et en puits jusqu'à $700 \mathrm{~m}$ de profondeur;

- des capteurs de déplacement ou de déformation.

Ce sont d'abord, dans trois forages, des inclinomètres en forage jusqu'à $700 \mathrm{~m}$ de profondeur maximale, ancrés dans le volcanisme et rendu solidaires des terrains par cimentation totale du puits. Pour la sur-

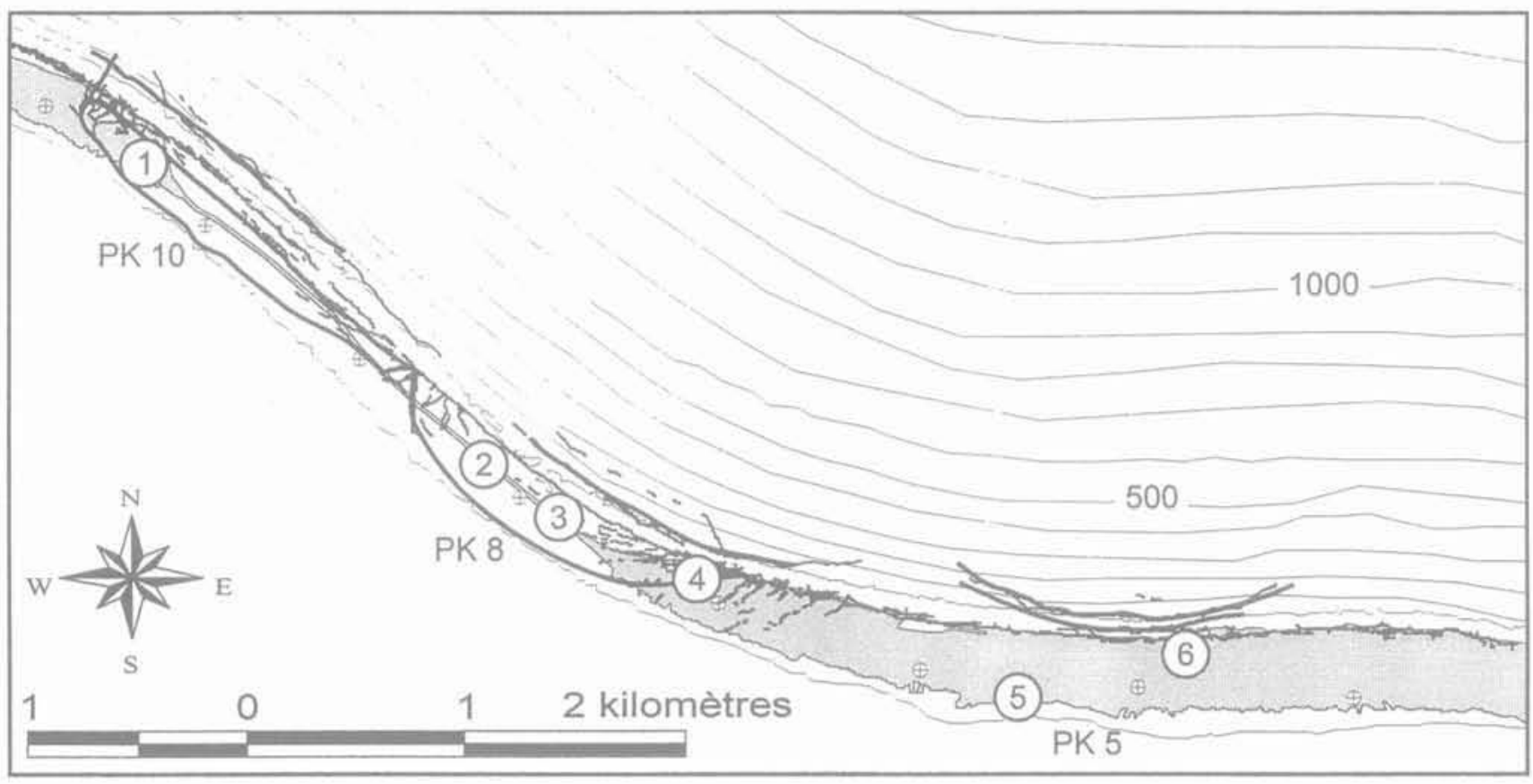

(1) GPS continu Inclinomètre Cable incliné Géophones

\section{(2) Inclinométre Cable incliné Géophones}
(4) Inclinomètre Cable horizontal Cable incliné

(6) GPS continu Cable incliné Géophones

FG.5 Carte de la zone Nord de la couronne corallienne. En traits gras: les limites des trois zones affectées (PK5, PK8, PK10). Les numéros renvoient à l'instrumentation réalisée à l'endroit considéré.

Map of the Northern area of the corallian rim. Bold lines: limits of the three affected zones (PK5, PK8, PK10). The numbers refer to the instrumentation installed at the considered location. 


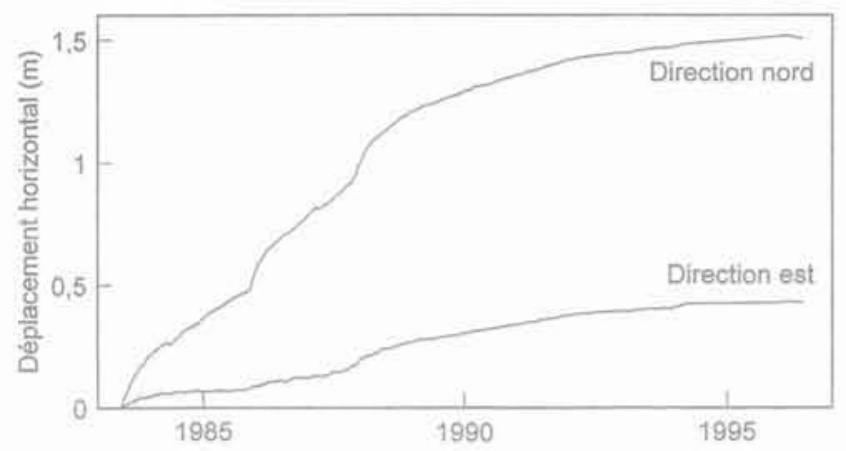

मiG.6. Les deux composantes du déplacement horizontal en fonction du temps au PK8 de la zone Nord de l'atoll. Ces mesures sont réalisées par géodésie.

The two components of the horizontal displacement as a function of time at PK8 of the atoll's Northern area. Goedetic measurements.

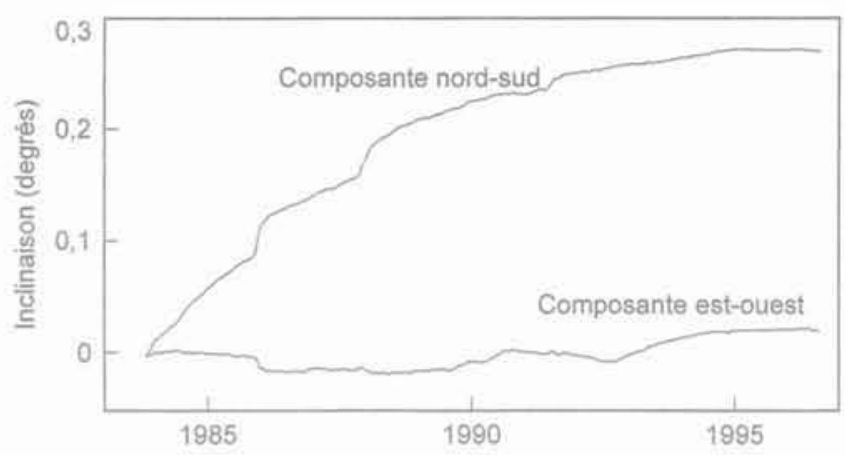

FIG. 8 Evolution en fonction du temps d'un inclinomètre situé dans les calcaires crayeux.

Evolution as a function of time of a clinometer located in the chalky limestones.

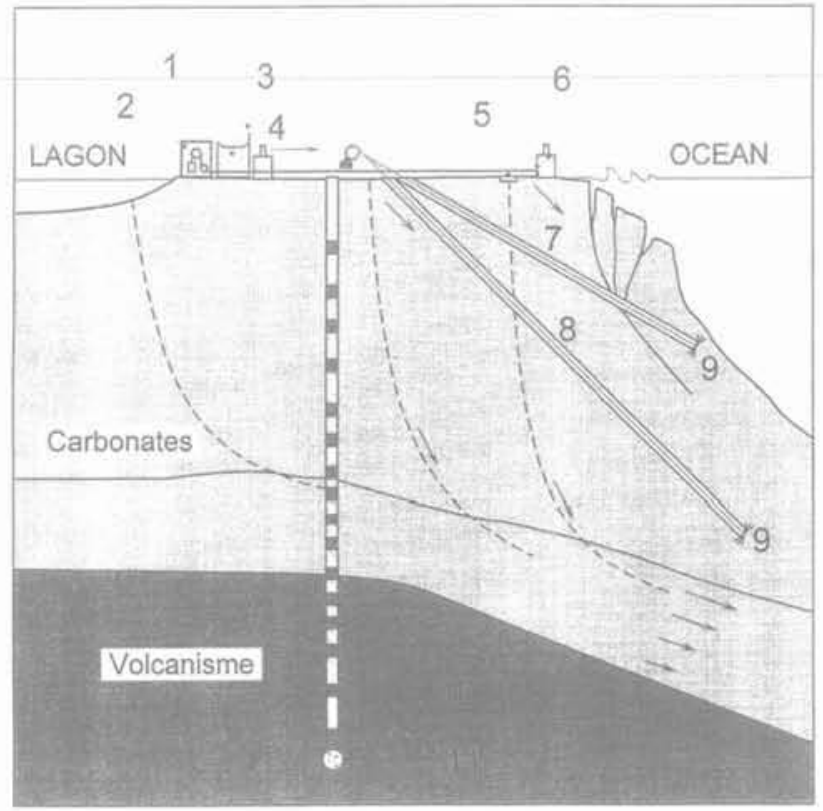

1 Route

2 Cáble tendu horizonta

3 Repere de nivellement

4 Pillier de visée

5 Faillemétre

6 Pilier jalon avec antenne GPS

7 Fil à $30^{*}$

8 Fill á $45^{\circ}$

9 Ancrage

10 Chaine d'inclinométres

11 Géphone tridirectionnel

FG.7 Coupe du bord de la couronne corallienne montrant Y'instrumentation réalisée in situ à partir de 1983.

Cross-section of the edge of the corallian rim, showing the instrumentation installed in situ from 1983 .

veillance des formations de pente les plus externes, des câbles tendus dans des forages obliques sont ancrés presque au débouché des forages dans l'océan. En surface, des câbles horizontaux tendus à travers la couronne corallienne permettent la surveillance, même en cas d'inaccessibilité de la couronne (en cas de crise par exemple). Enfin, les mesures géodésiques déjà mentionnées plus haut viennent compléter le dispositif. Depuis 1990, certaines de ces mesures sont effectuées par un système opérationnel de quatre balises GPS fonctionnant en permanence et en continu, et renvoyant leurs données en base arrière vers un système de traitement automatisé (Coulon, 1990; Flouzat, 1995).

Les mesures géodésiques de surface avaient montré l'évolution en surface de la couronne corallienne. Celleci est corroborée par les données de capteurs de pro- fondeur (Figs 8 et 9). L'inclinométrie, réalisée à partir de fin 1983, met en évidence un mouvement des masses sédimentaires carbonatées surplombant le volcan. Ce mouvement est régulier et localisé dans les couches calcaires présentant une structure poreuse développée (calcaires crayeux). La déformée en fonction du temps (Fig.9) met clairement en évidence la surface de découplage à une profondeur d'environ $400 \mathrm{~m}$, juste au-dessus du substratum volcanique qui, lui, reste stable. Le déplacement horizontal est plus fort au début des années 80 , et se ralentit fin des années 80 . Cette évolution est aussi visible sur la courbe de l'inclinomètre situé au niveau des calcaires crayeux à $400 \mathrm{~m}$ de profondeur. Les câbles tendus en forage inclinés (FIL) montrent une évolution semblable, confirmant que le mouvement affecte les formations de pente. 


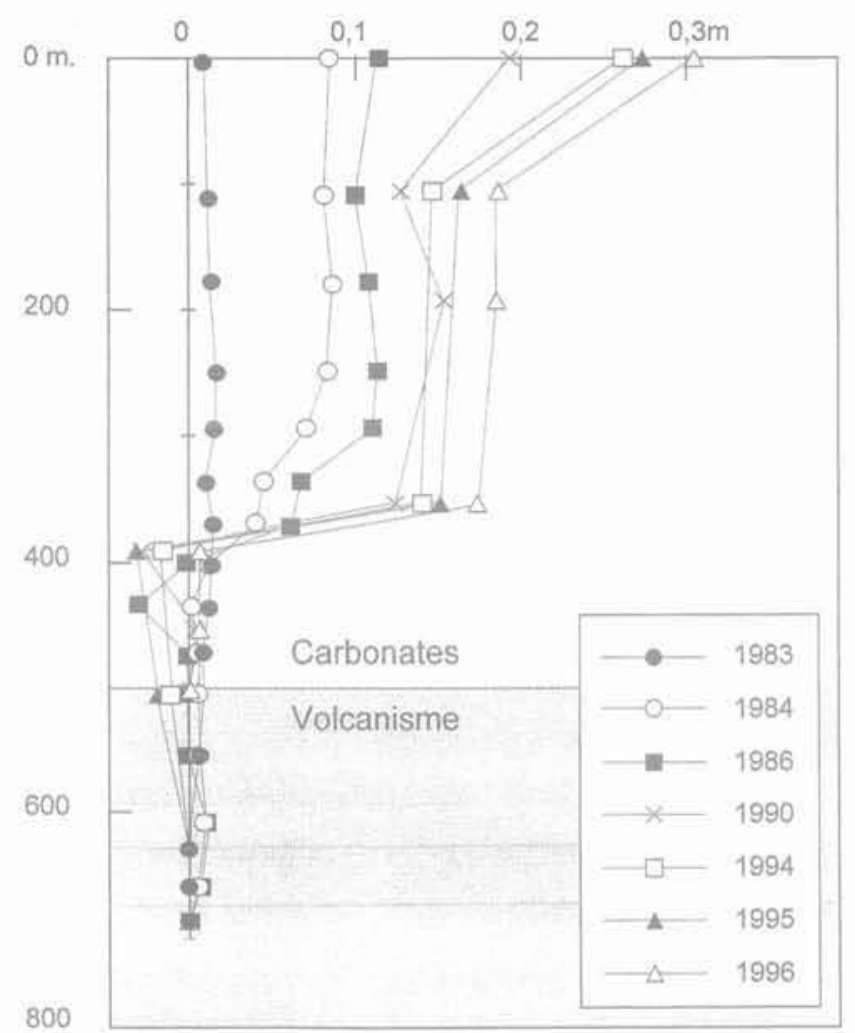

FIG. 9 Déformée reconstituée à partir des mesures de la chaine d'inclinométrie au PK8. On remarque clairement la zone de découplage vers $-400 \mathrm{~m}$ dans les calcaires crayeux et le comportement en bloc des formations situées au-dessus. Le volcanisme n'est affecté d'aucun mouvement.

Distortion reconstructed from measurements of the tiltmetry system at PK8. We can clearly see the decoupling zone near $-400 \mathrm{~m}$ in the chalky limestones and the block behavior of the formations located above that level. The lavas are not affected by any movement.

L'activité microsismique s'est manifestée dès le début aux frontières les plus externes du mouvement et de façon diffuse au sein de la masse rocheuse (Fig. 10), mettant en évidence un compartimentage en trois zones telles que décrit plus haut, les frontières extrêmes des zones en mouvement étant les plus visibles. En profondeur la distribution est limitée aux masses carbonatées, sans affecter le soubassement volcanique. Au début des années 80 les événements ont des magnitudes locales comprises entre 1 et 3 (Fig. 11), à l'exception d'un seul événement de magnitude 3,3 . Plus tard tous les événements détectés deviennent très faibles, avec une magnitude presque toujours comprise entre -1 et 1,5 et des moments sismiques correspondants compris entre $10^{9}$ et $10^{12} \mathrm{~N} . \mathrm{m}$. Les fréquences de coin sont comprises entre 3 et $17 \mathrm{~Hz}$. On peut estimer que l'événement moyen correspond à une source d'une taille de quelques dizaines de mètres avec un déplacement associé millimétrique, La figure 11 montre que l'essentiel de l'énergie sismique a été relâchée en 1980, année de la réalisation des derniers essais dans cette zone. Après, l'énergie se relaxe régulièrement, même si les sollicitations sismiques dues à la réalisation d'essais dans d'autres zones entraînent une légère augmentation de la relaxation sismique en zone Nord.

\section{1 .3}

\section{Géométrie, volume et origine de l'initiation du mouvement}

L'ensemble des mesures réalisées permettent de définir la géométrie et le volume des formations affectées par le mouvement. Des trois zones précitées, la zone "Camélia», située entre les PK 7 et 9 est la zone principale. Elle traverse la couronne jusqu'au lagon, alors qu'en «Françoise $»$ le mouvement n'affecte que partiellement la couronne, et qu'en "Irène» le mouvement n'affecte pas la partie émergée de la couronne mais seulement quelques dizaines de mètres d'épaisseur sous le niveau de l'océan. Les mesures inclinométriques montrent l'existence d'une surface de découplage au niveau des calcaires crayeux, c'est-à-dire vers $400 \mathrm{~m}$ de profondeur à la limite entre les carbonates et le substratum volcanique. Ceci est conforté par la distribution de la microsismicité. Le mouvement lent qui est mesuré correspond donc à un mouvement de fluage sur environ une centaine de mètres au niveau de la couche de calcaires craveux. Cette couche est surmontée de formations plus cassantes se comportant de façon plutôt rigide.

De plus, l'étude des fractures superficielles et de celles recoupées dans les forages obliques permet de délimiter subverticalement à l'intérieur d'une même zone plusieurs compartiments allant du plus externe côté océan au plus interne côté lagon (Fig. 7). Le volume maximal de la zone ( Camélia ») est estimé à $0,6 \mathrm{~km}^{3}$, mais le découpage en compartiments fait que le volume le plus affecté par le mouvement est constitué du compartiment le plus externe d'un volume de $0,04 \mathrm{~km}^{3}$.

Une modélisation analogique a été réalisée sur maquette à partir de plasticine pour la zone plastique et de sable pour le reste des formations carbonatées. A partir d'une reproduction à échelle réduite des volumes respectifs des unités mécaniques, on retrouve la distribution géométrique des déformations observée sur site ainsi que les caractéristiques de la tectonique gravitaire, en particulier les fractures de tête. Enfin, une modélisation numérique en dynamique permet de comprendre le phénomène à l'origine du mouvement. On a modélisé les effets d'une expérimentation libérant une énergie de $8 \mathrm{kt}$ réalisée dans le basalte à 800 mètres de profondeur. Les contraintes dynamiques engendrées entraînent la déformation plastique des formations les moins résistantes mécaniquement, qui correspondent aux calcaires crayeux. C'est ce phénomène qui est à l'origine de l'initiation du mouvement. On peut néanmoins penser que l'existence de fractures bordières naturelles avant la réalisation des essais correspond à une évolution naturelle des calcaires crayeux sous la charge de la masse corallienne. Les essais auraient donc fortement accéléré un phénomène naturel.

\section{1 .4}

\section{Réaction mécanique des formations de pente aux essais réalisés depuis 1980}

Une fois le mouvement amorcé en 1980, les essais réalisés ultérieurement dans le volcan sous le lagon à une distance de plusieurs kilomètres, ont encore un léger effet sur le mouvement de pente. C'est ce que montrent les figures 5 et 8 .

La modélisation numérique permet de comprendre l'origine de cet effet légèrement accélérateur (Fig. 12). La 

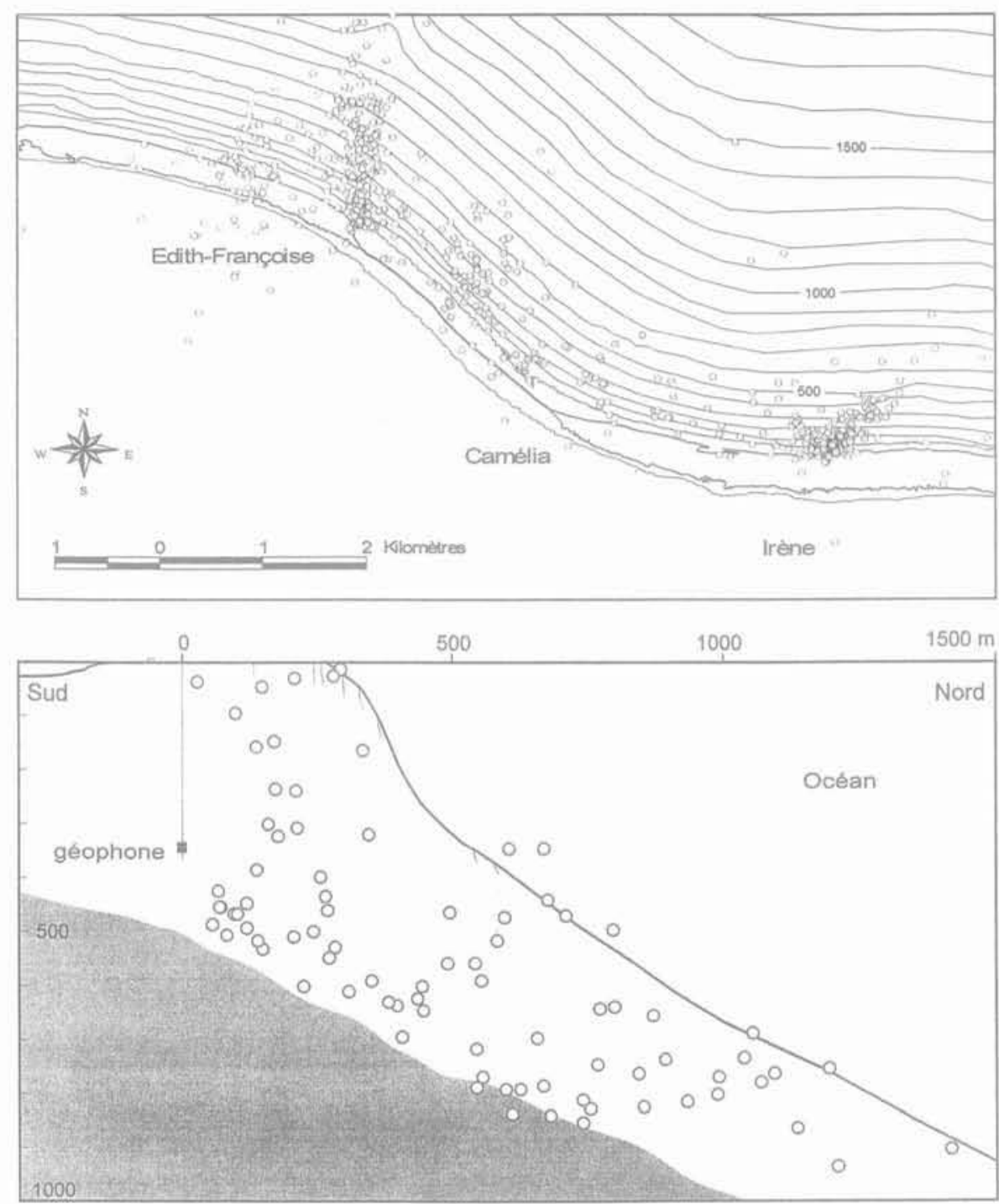

FG. 10 Sismicité de la zone Nord de la couronne corallienne, cumulée sur seize ans. Les zones les plus sismiques sont les frontières les plus externes du mouvement. Les microséismes sont situés dans la partie corallienne (gris clair) et non dans le volcanisme (gris foncé). Quelques événements localisés dans I'océan donnent une idée de la précision de la localisation.

Seismicity of the Northern area of the corallian rim accumulated over 16 years. The most seismic areas are the most extreme borders of the movement. The microseisms are located in the corallian part (light gray) not in the lavas (dark gray). A few events located in the ocean give an idea of the precision of localization.

propagation élastique des ondes générées par l'explosion (simulation aux nombres d'ondes discrets) est calculée depuis une source représentative d'un essai sous lagon de $65 \mathrm{kt}$ à $10 \mathrm{~km}$ (Crusem, 1986; Crusem et Caristan 1992). Puis, dans la zone de pente, le calcul non linéaire est effectué en prenant en compte le comportement viscoplastique avec cohésion de la craie. La pression interstitielle est calculée en tenant compte des échanges au niveau de l'interface massif-océan (Mariotti, 1990).

La figure 12 montre la distribution de la pression interstitielle résiduelle 100 s après l'essai. L'évolution de la vitesse matérielle au sein des couches crayeuses, en fonction du temps, est représentée sur la figure 13. Partant d'une vitesse de fluage nulle avant essai, on y voit bien l'accélération correspondant au passage des ondes sismiques $3 \mathrm{~s}$ après l'essai et à la montée de la pression interstitielle, puis la diminution de la vitesse avec la diminution de la pression interstitielle par diffusion et, enfin, le retour au bout de quelques mois à une vitesse très faible. Cette réponse temporelle des formations de pente correspond tout à fait aux observations faites in situ. Ainsi le comportement mécanique se révèle-t-il sensible à deux paramètres principaux caractérisant un essai donné: la distance à la zone «Camélia » et l'énergie réalisée.

Depuis 1980 plus de 80 essais ont été réalisés sur d'autres zones de la couronne et en lagon. Les mesures réalisées in situ à partir de fin 1983 ont mis en évidence expérimentalement la sensibilité du mouvement à l'effet des ondes sismiques provenant des essais réalisées à partir de 1981 dans d'autres zones de l'atoll. On peut observer sur ces courbes qu'il n'y a pas d'effet spécifique notable lié à la dernière campagne d'essais en 1995-1996. 


\section{Energie (Joules)}

Energie sismique cumulée
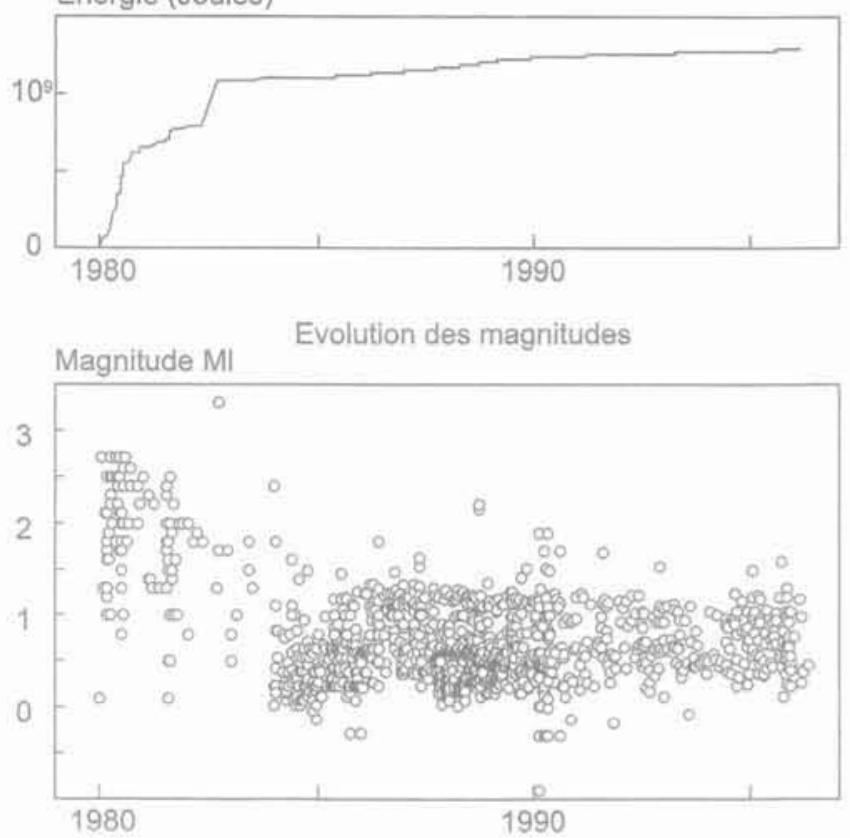

FIG.11 Énergie sismique et magnitude locale des événements en fonction du temps.

L'essentiel de l'énergie sismique est libérée au début des années 80 .

immédiatement après les essais réalisés dans la zone. L'augmentation apparente du nombre d'événements en 1983-1984 est due à l'installation des géophones à $700 \mathrm{~m}$ de profondeur.

Seismic vield and local magnitude of the events as a function of time. The most part of the seismic yield is released in the early $80 \mathrm{~s}$, immediately after the tests conducted in the area, The apparent increase in the number of events in 1983-1984 is due to the installation of geophones at a depth of $700 \mathrm{~m}$.

En plus de l'approche numérique présentée ci-dessus, une approche semi-empirique, basée sur les lois de fluage déterminées en laboratoire et l'observation in situ de la réponse temporelle de la masse sédimentaire a permis, à partir de 1986, de faire des prévisions du comportement global des zones en mouvement en fonction des campagnes programmées, de la position et de l'énergie de chaque essai. Sur l'ensemble des essais réalisés durant une dizaine d'années jusqu'en 1996, les effets mesurés a posteriori sont toujours restés dans les marges prévisionnelles.

Le comportement mécanique de la zone Nord de l'atoll de Mururoa a donc été un des éléments dimensionnants quant aux énergies réalisables au Centre d'Expérimentation du Pacifique, et à leur positionnement dans l'atoll.

\section{$3 i 15$}

\section{Le comportement à long terme de la zone Nord de Mururoa}

Les courbes des figures 6,8 et 11 montrent que l'évolution du mouvement depuis 1980 est globalement en perpétuelle décélération, abstraction faite de quelques accélérations limitées dans le temps et liées à des campagnes particulières.
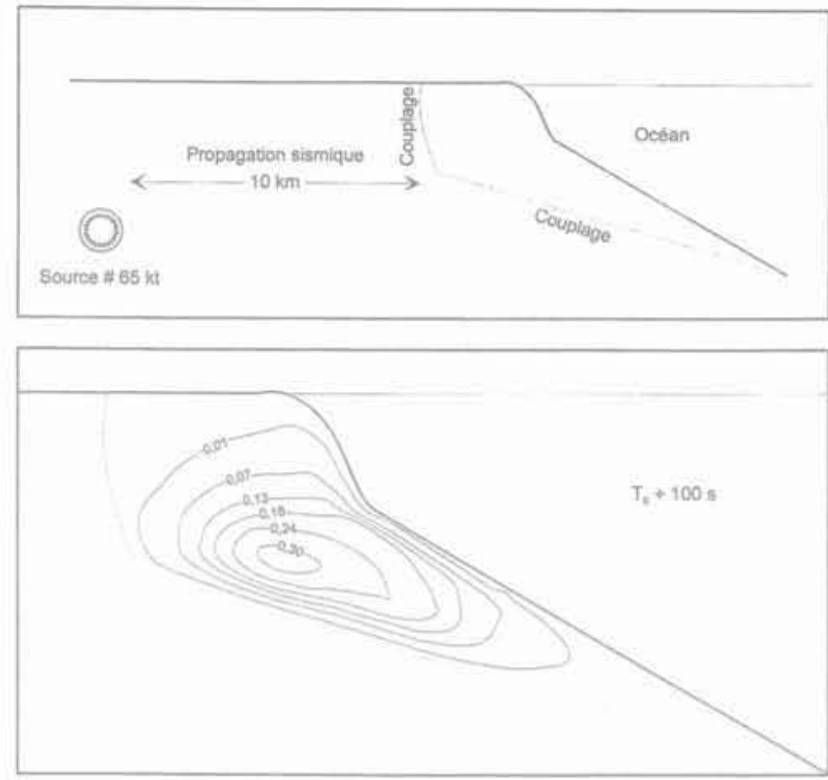

FG 12 Calcul du comportement mécanique de la zone Nord sous sollicitation sismique. En haut, schéma du couplage du calcul élastique de propagation depuis la source et du calcul mécanique non linéaire au niveau du tombant. En bas: pression interstitielle générée par l'ébranlement au temps $\mathrm{T}$ : $100 \mathrm{~s}$ après l'explosion.

Calculation of the mechanical behavior of the Northern area under seismic loading. Top: diagram of the coupling of elastic propagation design from the source, and non linear mechanical calculation at the rim. Bottom: interstitial pressure generated by disturbance at time T: 100 seconds after the burst.

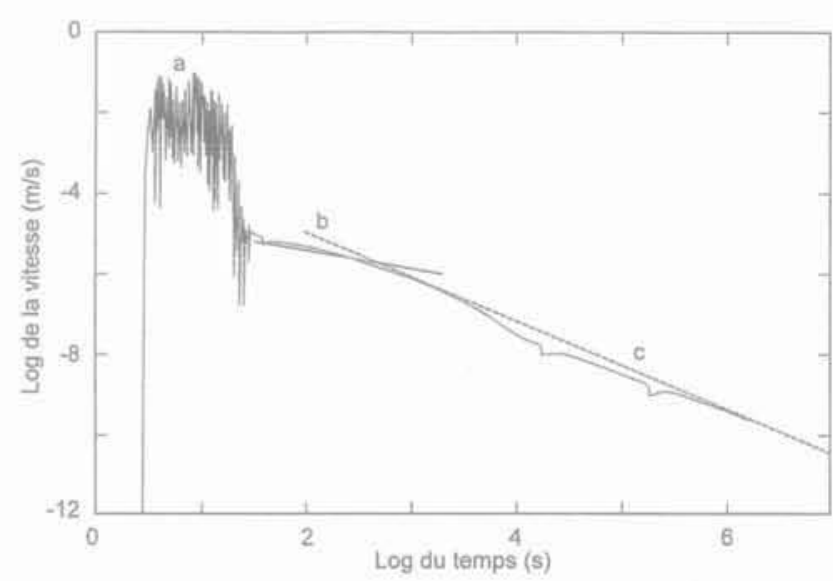

FG. 13 Calcul de l'évolution en fonction du temps d'un capteur numérique situé dans la couche de calcaires crayeux (voir Fig. 12). On distingue une phase dynamique avec le passage de l'onde (a), une phase de diffusion de la pression (b) puis une phase de fluage (c) correspondant à un retour à des vitesses de fluage très lentes.

Calculation of the evolution as a function of time of a digital sensor located in the chalky limestone layer (see figure 12). We can see a dynamic phase, with passage of the wave (a), a pressure diffusion phase (b) then a creep phase (c) corresponding to return to very slow creep rates. 

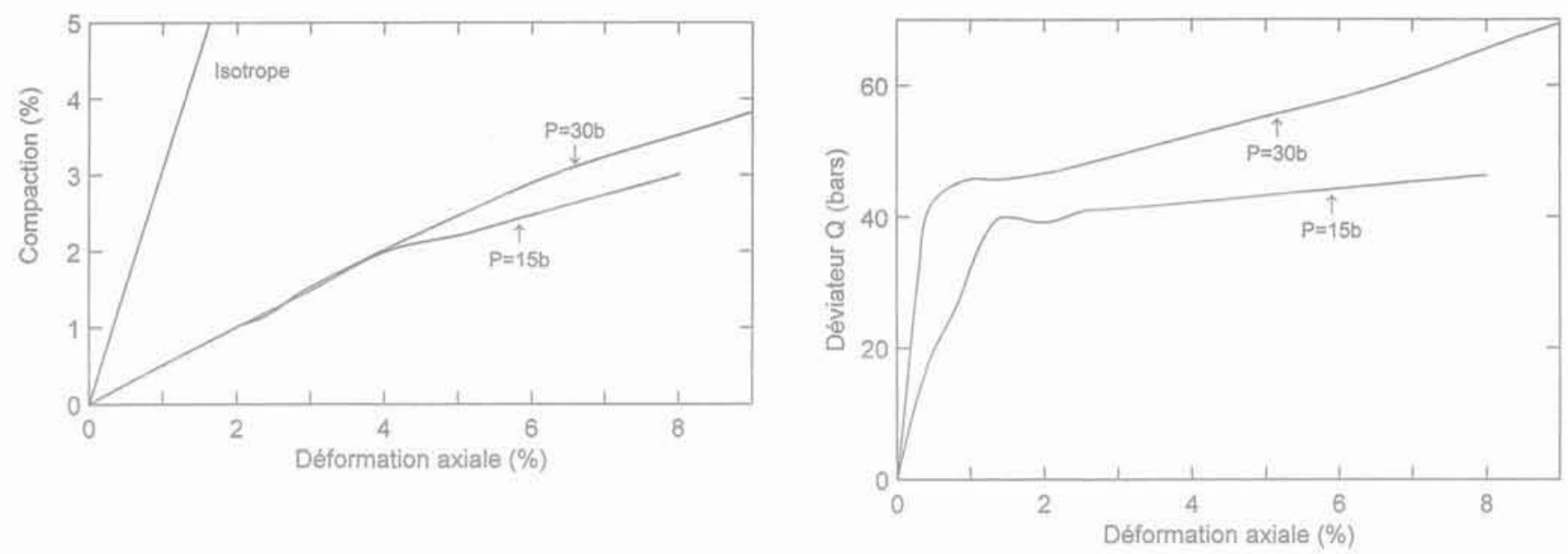

FiG. 14 Mesure en laboratoire des caractéristiques des calcaires crayeux; a) Compaction (déformation volumique) en fonction de la déformation axiale. On note le caractère compactant du matériau pour trois états de contraintes différents: une pression isotrope sans déviateurs, deux pressions de confinement différentes avec déviateur, b) Courbes contraintes-déformations associées.

Laboratory measurement of the characteristics of chalky limestones: a) Compaction (volumetric deformation) as a function of axial deformation. We can note the compacting character of the material for 3 different stress states : an isotropic pressure without deviators, two different confinement pressures with deviator, b) Stress/associated deformation curves.

Les caractéristiques mécaniques des calcaires crayeux, mesurées en laboratoire, montrent que ceux-ci sont compactants. Au cours de la déformation, le volume de la roche tend à diminuer (Fig. 14). Dans ce type de comportement les matériaux ne passent pas en général en fluage tertiaire. La diminution progressive

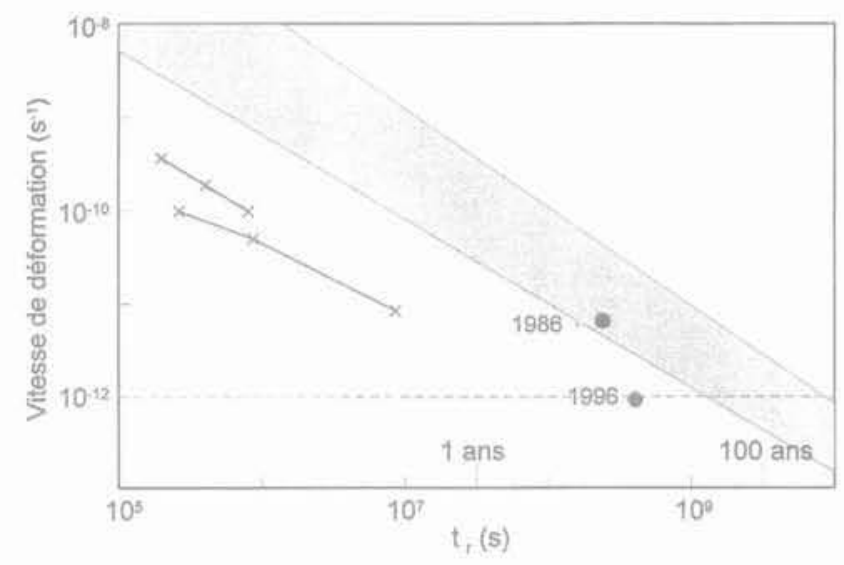

FIG. 15 En grisé : relation expérimentale entre le temps à la rupture et la vitesse de déformation minimum (déduite d'expériences sur des craies (Morlier, Saïto). Les croix représentent l'évolution de la vitesse au cours du temps lors des expériences réalisées en laboratoire sur les calcaires crayeux prélevés in situ, et les points l'état mesuré in situ par rapport à 1980 , date du début du mouvement.

Hatched area: experimental relationship between the time at failure and the minimum deformation speed (from experiments on chalks (Morlier, Saito)). The $(X)$ represent the evolution of speed in time during experiments conducted in the laboratory on chalky limestones sampled in situ, and the current situation as measured in situ relative to 1980 , date at which the movement started. des vitesses de fluage sous charge ne conduit pas à la rupture, ce que confirment des expériences menées en laboratoire à très faible vitesse de déformation (Fig, 15) sur les calcaires crayeux.

Saïto $(1961,1980)$ et Morlier (1966) ont réalisé des expériences sur des craies dans des domaines de vitesse de déformation beaucoup plus élevée, qui ont conduit au fluage tertiaire et à la rupture. Si l'on extrapole les lois phénoménologiques correspondantes reliant le temps écoulé depuis le commencement du fluage jusqu'à la rupture, on obtient la zone grisée de la figure 15. Les points mesurés in situ et les expériences conduites en laboratoire sur des échantillons de calcaire tendre ont montré une phénoménologie de fluage primaire voire secondaire sans atteindre la fluage tertiaire. Ainsi, les points correspondants sont situés en dessous de la zone grisée sur la figure 15. Néanmoins, sur cette figure, l'intersection de la vitesse de déformation actuellement mesurée in situ $\left(10^{-12} \mathrm{~s}^{-1}\right)$ avec la courbe de temps à la rupture conduirait à une rupture entre 100 et 1000 ans si cette vitesse était constante. Il est probable qu'elle continuera à s'amortir, ce qui conduirait alors à des temps encore plus longs, voire à une stabilisation. Compte tenu des remarques faites plus haut, on peut considérer que l'évolution la plus probable va vers une quasi-stabilisation du mouvement dans les décennies à venir.

En tout état de cause, un système de surveillance, appelé TELSITE, a été installé. Il reprend en les simplifiant les éléments essentiels du dispositif de 1983 et intègre sur place des moyens numériques de transmission, stockage et traitement sur place entièrement automatiques et alimentés par panneaux solaires. Aucune présence humaine n'est requise; les capteurs sismiques, GPS, inclinométriques sont traités, et les résultats envoyés quotidiennement par satellite au Département d'Analyse et Surveillance de l'Environnement du CEA/DAM qui effectue, depuis la France métropolitaine, une télésurveillance opérationnelle et technique. 


\section{Conclusion}

La présence de calcaires crayeux au niveau des formations de pente dans la partie Nord de la couronne corallienne de l'atoll de Mururoa a favorisé l'ouverture de certaines failles bordières lors des essais réalisés dans cette zone en 1979-1980. Une partie des formations de pente a été affectée d'un mouvement de subsidence continu et décroissant dans le temps en trois endroits de cette zone: PK5N, PK8N, PK10N. L'instrumentation réalisée en 1983 a permis de comprendre le comportement mécanique, de prévoir et de suivre la réaction aux sollicitations sismiques au cours de la réalisation de plus de 80 essais. L'évolution mécanique observée depuis plus de quinze ans, les expériences réalisées sur maquette et les propriétés mécaniques des calcaires crayeux, mesurées en laboratoire, conduisent à penser que le mouvement, dont la vitesse a diminué de plus d'un ordre de grandeur depuis 1980 et malgré les essais réalisés, devrait aller vers une stabilisation. En particulier la campagne 1995-1996 n'a eu aucun effet mécanique notable. En tout état de cause, un système de surveillance géomécanique automatique et téléopéré est installé et devrait fonctionner au moins pour les dix ans à venir.

\section{Bibliographie}

Bouchez J. Lecomte R. - Les Atolls de Mururoa et Fangataufa, les expérimentations nucléaires: effets mécaniques, lumino-thermiques et électromagnétiques, Masson, 189 p. 1995.

Bouchez J., Mariotti C. - « Différences rhéologiques sous pression de confinement entre des crajes tendres naturelles et synthétiques $x_{1}$, ISRM-SPE International Symposium, Pau 1989, Balkema, Vol. 2, p. 93-99, 1989

Buigues D., Gachon A., Guille G. - $\alpha$ L'atoll de Mururoa (Polynésie française) L - Structure et évolution géologique n. Bull. Soc. géol. France, t. 163, n 5, p. 645-657, 1992.

Buigues D. - «Mururoa and Fangataufa : sea-level changes, karstification and the atoll morphology» Bull. Soc, géol. France.

Coulon B., Caristan Y. - a Monitoring displacements by GPS: a calibration test 1 . Cahiers du Centre européen de géody. namique et séismologie, vol. 2, p. 253 261, 1990.

Crusem R., Caristan Y. - Moment tensor inversion, estimation and seismic coupling variability at the french Centre d'Expérimentation du Pacifique 1, Bull. Seism. Soc. Amer., n`3, p. 1253-1274, 1992.
Douglas B. - "Global sea level rise $n$, Journal of Geophysical Research, 96, p. 69816991. 1991.

Flouzat M., Fourmaintraux D, Camphuysen R. - «Advanced continuous monitoring of subsidence above gas fields using spatial geodetic measurements , $_{\text {, }}$ Proceedings of the International Symposium on Land Subsidence, The Hague, Netherlands, 16-20 october 1995. p. 269-280.

Guille G., Goutière G., Sornein J.-F; - Les Atolls de Mururoa et Fangataufa : géologie, pétrologie et hydrogéologie, Masson, 167 p.. 1993.

Guille G., Maury R.C. Buiques D. Bellon H., Gachon A., Caroff M. - $\alpha$ L'atoll de Mururoa (Polynésie française) III Conclusions générales», Bull. Soc. géol. France, t. $163, n^{\circ} 5$, p. 681-685, 1992.

Habib P, - « Aspects géotechniques de I'accident du nouveau port de Nice 1 , Revue Française de Géotechnique, $\mathrm{n}^{\circ} 65$, p. 3-15, 1994.

Heezen B.C. Ewing M. - "Orlèansville earthquake and turbidity currents $p$, Bull. of the American Association of Petroleum Geologists, vol. $39, n^{\circ} 12$, p. 2505-2514, 1992
Jansen E. Befring S., Bugge T, et al. "Large submarine slides on the Norvegian continental margin : sediments, transport and timing.1, Marine Geol., vol. 78 , p. $77-107,1987$

Kulikov E.A., Rabinovich A.B., Thomson R.E., Bornhold B.D. - "The landslide tsunami of November 3, 1994, Skagway Harbor, Alaska \#, J. of Geoph. Res. Oceans, vol. 101 (C3), p. 6609-6615, 1996.

Moore J.G. - « Giant submarine landslide on the Hawaiian Ridge D, Geologica! Survey Research, 501-D, p, D95-D98. 1964

Morlier P. - $\alpha$ Le fluage des roches $\nsim$. Annales de I'TTBTP n²17, 1996 .

Murty T.S. - - Submarine slide-generated water waves in Kitimat Inlet, British Columbia, J. of Geoph. Res., vol. 84 (C12), p. 7777-7779, 1979.

Saito M. Uezawa H. - «Failure of soil due to creep 3, 5th ICS MFE, Paris, vol. 1. p. $315-318,1961$

Saito M - is Semi logarithmic representa. tion for forecasting slope failure w, Proc 3rd International Symp. on Landslides. New Dehli, 1, p. 321-324, 1980.

Voight B. - Rockslides and avalanches, vol. 1. Elsevier, p. 833, 1978. 